\title{
Extraction of group index of lossy photonic crystal waveguides
}

\author{
Amrita Debnath, Kapil Debnath,* Liam O'Faolain \\ SUPA, School of Physics \& Astronomy, University of St Andrews, North Haugh, St Andrews KY16 9SS, UK \\ *Corresponding author: kd343@st-andrews.ac.uk
}

Received Month X, XXXX; revised Month X, XXXX; accepted Month X, XXXX; posted Month X, XXXX (Doc. ID XXXXX); published Month X, XXXX

\begin{abstract}
We present a numerical approach to extract group index in photonic crystal waveguides using two and three-dimensional finite-difference time-domain methods and make a quantitative study of the effects of loss on slow light propagation in photonic crystal waveguides. Photonic crystal waveguides are simulated with varying material loss and varying photonic crystal waveguide length. Finally we validate our method by comparing three-dimensional simulation results with experimental results. (C) 2013 Optical Society of America

OCIS Codes: (140.3490) Lasers, distributed-feedback, (060.2420) Fibers, polarization-maintaining, (060.3735) Fiber Bragg gratings, (060.2370) Fiber optics sensors.

http://dx.doi.org/10.1364/OL.99.099999
\end{abstract}

The concept of slow light in photonic crystal (PhC) waveguides has been explored over the last decade both from a fundamental point of view and for practical applications. Slow light $\mathrm{PhC}$ waveguides have been used for applications such as optical delay lines [1] and enhanced light matter interaction both for linear [2] and nonlinear [4-5] applications.

Planar $\mathrm{PhC}$ waveguides are generally realised by creating a line defect in the $\mathrm{PhC}$ lattice and are commonly known as the $\mathrm{W} 1 \mathrm{PhC}$ waveguide [6]. Interest in $\mathrm{PhC}$ waveguides arises from the fact that the group index can be very large near the band edge of its dispersion relation, which results in slowing down of optical pulses. However, simple W1 waveguides suffer from high group velocity dispersion close to the bandedge. This is very detrimental for many applications, causing, for example, pulse broadening. To overcome this problem, $\mathrm{PhC}$ waveguide designs have been proposed and realised in which the band structure is modified, creating a flat group velocity curve around the operating wavelength $[7,8]$. While these designs have enabled a host of key results [9] it has been realised that optical loss has a serious effect on slowlight, placing limits on the values that may be attained [10]. In this paper, we quantify the effects of loss on slow light propagation and describe a powerful new approach to model slow light photonic crystals.

The Finite Difference Time Domain (FDTD) method is a very powerful numerical technique for modelling optical components. In the past, FDTD has been used to calculate the dispersion relation of $\mathrm{PhCs}$, as well as estimating transmission behaviour of $\mathrm{PhC}$ waveguides; however obtaining information on the group index is problematic. Time of flight methods have been used but are computationally intensive due to the need to make simulations at a large number of wavelengths [11]. Plane wave expansion methods, in which the group index is determined from the band structure are most popular but essentially use an idealised device and cannot determine the group index for lossy (realistic) systems. Here we propose and demonstrate a new method for calculating the group index spectrum using the FDTD method. By deliberately introducing material loss, we can extract the group delay from the resulting change in transmission providing insights into the effects of loss on slow light waveguides and a simple and effective method for determining the group index.

In this work, all the simulations were carried out using the RSOFT Fullwave module, commercially available software for FDTD analysis. The waveguide structure is shown in figure 1 . The W1 waveguide is defined in a triangular lattice of air holes in silicon by removing one row of holes from the lattice. To launch and measure the light, source and monitor are placed on the access waveguides on either side of the $\mathrm{PhC}$ waveguide. For the initial studies we made a 2D FDTD analysis. The lattice constant (a) and the hole radius ( $\mathrm{r}$ ) are chosen to be $414 \mathrm{~nm}$ and $0.29 \mathrm{a}$ respectively. The length of the W1 waveguide is $40 \mu \mathrm{m}$. The effective index of $\mathrm{Si}$ is chosen to be 2.7 at $\lambda=1550 \mathrm{~nm}$. Within the wavelength range of interest, the waveguide exhibits a single TE polarized (dominant electric field is in the plane of $\mathrm{PhC}$ ) mode. In order to calculate the group index curve, we first calculate the transmission spectrum of the waveguide, shown as the red curve in figure 1 . We then calculate the transmission spectrum of the waveguide after increasing the material loss ( $\mathrm{PhC}$ region only) (related to the approach of [12]), shown as the green curve in figure 1. It is quite apparent from the two spectra that the deviation between them increases as the band edge is approached. For a given wavelength, the transmitted intensity in two cases can be written as follows:

$$
\begin{aligned}
& T_{1}=T_{0} e^{-\alpha l} \\
& T_{2}=T_{0} e^{-\left(\alpha+\alpha_{a b s} l\right)}
\end{aligned}
$$

where $\mathrm{T}_{0}$ is the intensity of the launched light, $\mathrm{T}_{1}$ is the intensity of the transmitted light and $\mathrm{T}_{2}$ is the intensity when additional loss has been introduced. In these equations only the losses incurred in the $\mathrm{PhC}$ region are 
considered, and losses due to the access waveguides and coupling losses are ignored since they are common to both cases. $\alpha$ is the attenuation coefficient, which might include scattering losses, free carrier absorption and so on, of the device being studied and $\alpha_{a b s}$ is the absorption coefficient introduced via material absorption and used to probe the group delay. $\mathrm{l}$ is the length of the $\mathrm{PhC}$ waveguide.

Following [6], for low material loss the absorption coefficient varies linearly with the material extinction coefficient and can be approximated as:

$$
\alpha_{a b s}=\frac{4 \pi n_{g} f}{\lambda_{0} n}(\kappa+\Delta \kappa)
$$

where $\mathrm{n}_{\mathrm{g}}$ is the group index, $\mathrm{f}$ is the fractional electric field energy inside the silicon, $\mathrm{k}$ is the material extinction coefficient) $\Delta \mathrm{k}$ is the introduced material extinction coefficient), $\lambda_{0}$ is the free space wavelength and $n$ is the refractive index of silicon. Rearranging equations (1)-(3), we can write the group index as:

$$
n_{g}=\frac{\lambda_{0} n}{4 \pi f \Delta \kappa l} \ln \left(\frac{T_{1}}{T_{2}}\right)
$$

For our analysis we have chosen $\lambda_{0}$ at $1550 \mathrm{~nm}$ and using $2 \mathrm{D}$ mode analysis we found that $\mathrm{f}$ is $92 \%$ around the operating wavelength. Here we used $\Delta \mathrm{k}=0.0001$. The calculated group index spectrum is shown in blue in figure 1. The spectrum shows the expected rapid increase in

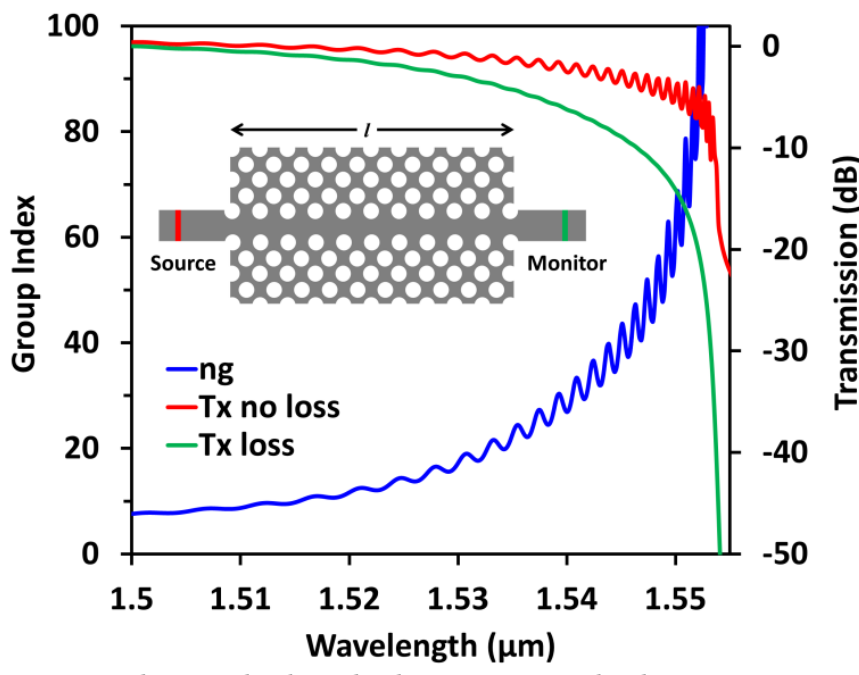

group index as the band edge is approached.

Fig. 1. Simulated transmission spectra and calculated group index spectrum for a W1 waveguide (schematic shown in the inset).

In an ideal, lossless photonic crystal, the group index goes to infinity as the photonic band edge is approached; however, it has been shown that the presence of loss can strongly modify the dispersion curve [13]. Pedersen et al [10] have identified the limits imposed on the maximum attainable group index by such loss induced modifications and described the effect as a "smearing" of the photonic density of states. In figure 2 , we indeed observe a divergence between the group index curves calculated in the presence of loss and that calculated for lossless case as the band edge is approached. It should be noted that we have chosen to use the richer group index curve of dispersion engineered $\mathrm{PhC}$ waveguides [8] for this demonstration. As the loss increases, the maximum group index reached drops noticeably in good qualitative

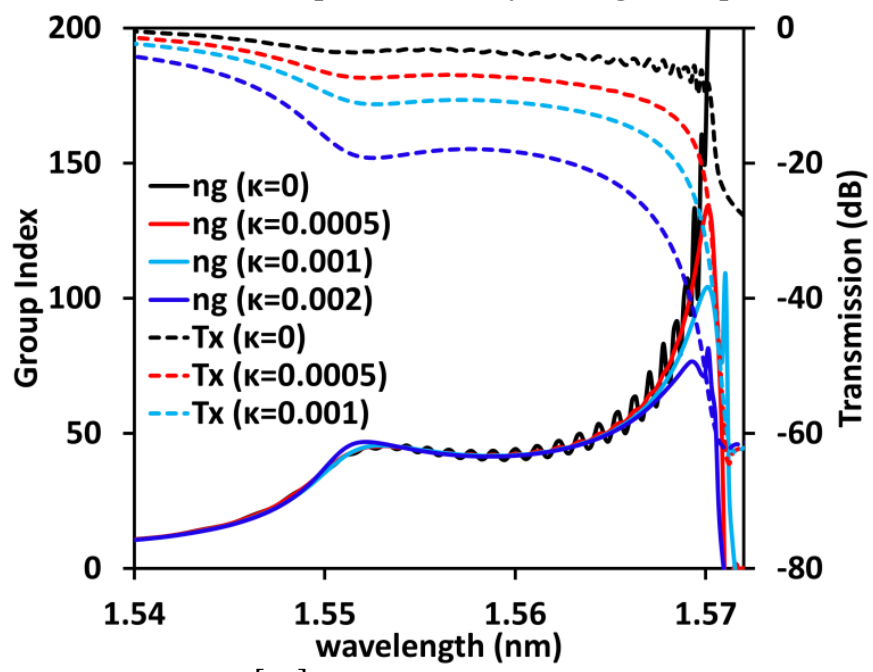

agreement with [10].

Fig. 2. Transmission spectra (dashed line) and corresponding group index spectra (solid line) with varying material loss ( $\mathrm{k}$ ) for a dispersion engineered $\mathrm{PhC}$ waveguide. For all the cases the $\Delta \mathrm{k}$ value was 0.0001 . We follow the approach proposed in [Li2008] to achieve flat band response, by shifting the two rows of holes adjacent to the center of the waveguide by $\mathrm{s} 1=0.12 \mathrm{a}$ and $\mathrm{s} 2=-$ 0.08 a respectively.

We find, for example, that the group index has a maximum value of $\sim 100$ for $\mathrm{k}=0.001$, which corresponds to a loss of around $20,000 \mathrm{~dB} / \mathrm{cm}(\mathrm{Eq} 3)$. Figure 2 clearly shows a limiting group index for each $\mathrm{k}$ values indicating that there is a threshold value of the $\mathrm{n}_{\mathrm{g}}-\mathrm{k}$ product; above which the smearing of the optical density of states becomes important [10]. Importantly, this value is orders of magnitude greater than that encountered in state of the art devices [14].

To further investigate this,,, we have calculated group index spectra with different probing material losses $(\Delta \mathrm{k})$ and $\mathrm{PhC}$ lengths (1). The transmission spectra and the group index curves are shown in figure 3 . Figure 3 a shows the transmission spectra with and without material losses and its corresponding group index curves. Figure $3 \mathrm{~b}$ shows the transmission spectra for device length of $40 \mu \mathrm{m}$ and $80 \mu \mathrm{m}$ and corresponding group index curves. It is clear from the group index spectra that these material losses and device lengths have no appreciable effect on the calculation. 
To further verify our method, we compared the experimentally measured group index curve of a fabricated $\mathrm{PhC}$ waveguide with group index curves calculated using 3D FDTD and plane wave expansion (PWE) method. For the experimental demonstration we choose a triangular $\mathrm{PhC}$ with lattice period of $\mathrm{a}=420 \mathrm{~nm}$ and hole radius of $r=114 \mathrm{~nm}$ in a $220 \mathrm{~nm}$ layer of silicon suspended in air. We considered an engineered $\mathrm{PhC}$ waveguide with s1 and s2 of $50 \mathrm{~nm}$ and $-16 \mathrm{~nm}$ respectively. For the FDTD calculation we choose the refractive index of silicon to be 3.47, the device length was $20 \mu \mathrm{m}$ and the material loss $\mathrm{k}=0.001$. For the $3 \mathrm{D} \mathrm{PhC}$ waveguide, the fractional electric field energy inside the silicon is found to be $95.6 \%$. The fabrication of the $\mathrm{PhC}$ waveguides started from a silicon-on-insulator substrate with $220 \mathrm{~nm}$ of top silicon layer with $2 \mu \mathrm{m}$ of buried oxide.

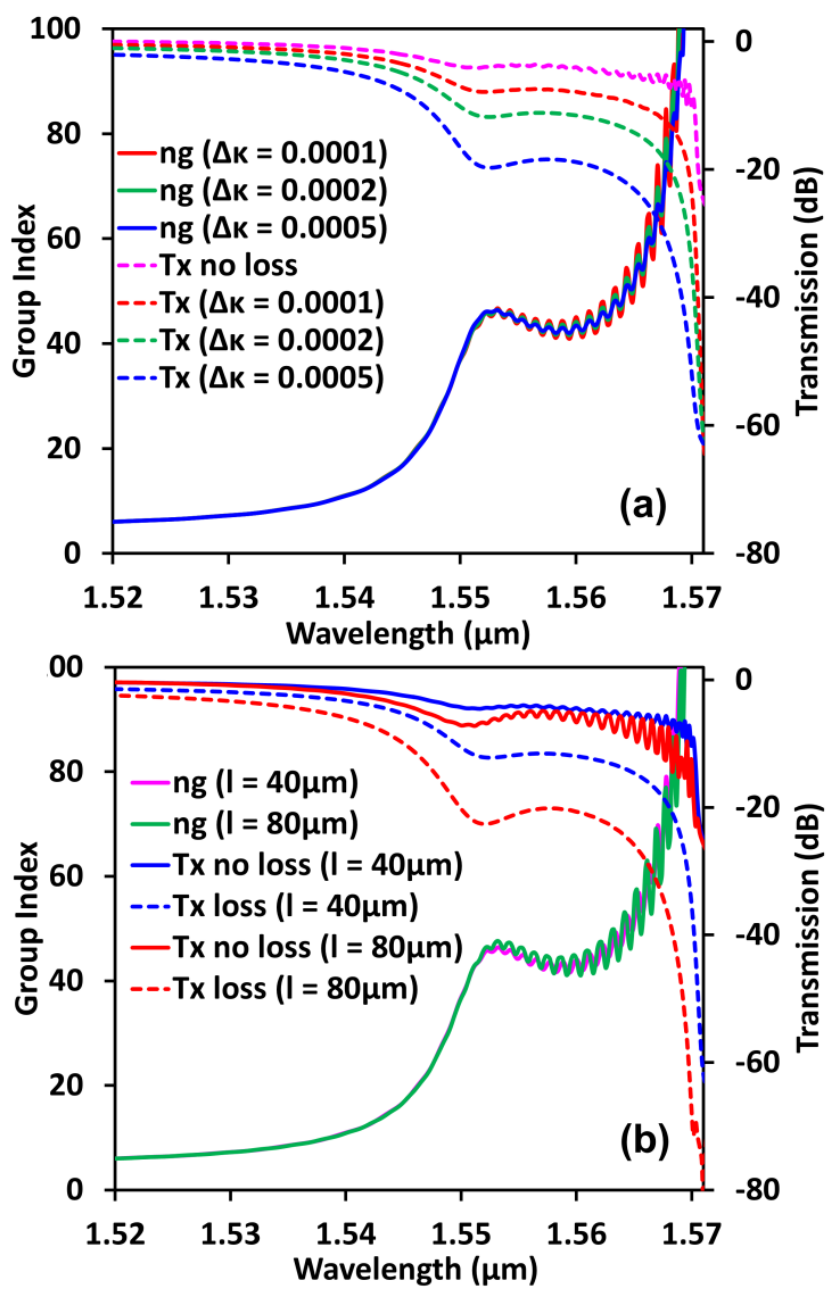

Fig. 3. (a) Transmission spectra and corresponding group index spectra with varying material loss $\left(\Delta_{\mathrm{K}}\right)$. (d) Transmission spectra and corresponding group index spectra with varying length of $\mathrm{PhC}$ waveguide

The $\mathrm{PhC}$ patterns were transferred into the silicon layer using ebeam lithography and subsequent etching using reactive ion etching (RIE). Details of the fabrication steps can be found in [15]. To create the suspended membrane of the $\mathrm{PhC}$ waveguide, the buried oxide underneath the $\mathrm{PhC}$ was selectively removed by wet etching with hydrofluoric acid. Finally, the sample was cleaved to form optical facets for measurement using the end-fire technique. We used a similar technique as reported in [16] to measure the group index curve of the fabricated $\mathrm{PhC}$ waveguides. Figure 4 shows the experimental (green curve) and calculated group index curves using 3D FDTD (green) and PWE (black) methods. There was a $5 \mathrm{~nm}$ and $15 \mathrm{~nm}$ shift between the experimental and 3D FDTD curves and PWE curves respectively, as slight variation between designed and fabricated hole size is unavoidable. It is clear from Fig. 5 that three-dimensional FDTD results are in good agreement with experimental results. Here one can notice fringes occurring in the calculated group index curve, which arises from the fringes in the transmission spectra (also noticeable in figures 1,2 and 3). These fringes are caused by the Fabry-Perot (FP) cavity formed at the interfaces between the $\mathrm{PhC}$ waveguide and the access waveguides. For example, consider a cavity with length $20 \mu \mathrm{m}$ and a group index of 40 . In this case the expected fringe spacing is $\sim 3 \mathrm{~nm}$. This agrees with the $\sim 3 \mathrm{~nm}$ fringe spacing observed in figure $4 \mathrm{~b}$ at a wavelength of $1595 \mathrm{~nm}$. It should be noted that the fringe spacing is larger in the simulated case as the cavity length is much shorter than that of the fabricated sample.

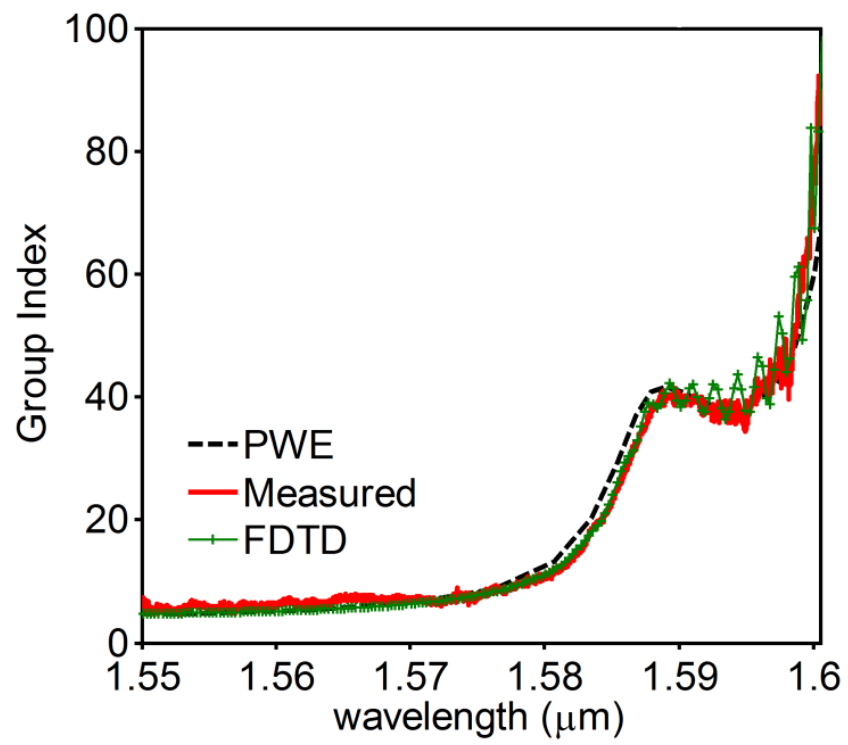

Fig. 4. Group index spectra for an engineered $\mathrm{PhC}$ waveguide, with $\mathrm{s} 1=50 \mathrm{~nm}, \mathrm{~s} 2=-16 \mathrm{~nm}$, measured (red solid line), calculated using PWE method (balck dashed line) and using FDTD method (green solid star line).

In conclusion, we have carried out a numerical study to demonstrate how the group index of $\mathrm{PhC}$ waveguides can be extracted from their transmission spectrum using the FDTD method. The 2D simulations are carried out for varying material loss and varying length of the $\mathrm{PhC}$ device for both $\mathrm{W} 1$ and dispersion engineered $\mathrm{PhC}$ waveguides. We have accessed regimes of high group index and high optical loss that are not experimentally accessible and quantified the limits on slowlight due to loss induced smearing of optical density of states. For the group index and loss values typically encountered in state of the art devices, we find excellent agreement between simulated and experimental results making our approach 
an excellent tool for the design of slow light photonic crystal waveguides.

This work is supported by an EPSRC Impact Acceleration Grant and by a European Research Council Starting grant (no. 337508). The authors would like to thank Tom P White and Lucio C. Andreani for useful discussion and suggestions.

References

1. R. W. Boyd, D. J. Gauthier, and A. L. Gaeta, Opt. Photon. News 17(4), 18 (2006).

2. 2. H. C. Nguyen, S. Hashimoto, M. Shinkawa, and T. Baba, Opt. Express 20, 22465 (2012).

3. 3. M. Soljacic and J. D. Joannopoulos, Nat. Mater. 3, 211 (2004).

4. 4. J. Li, L. O'Faolain, and T. F. Krauss, Opt. Express 20, 17474 (2012).

5. R. S. Jacobsen, K. N. Andersen, P. I. Borel, J. Fage Pedersen, L. H. Frandsen, O. Hansen, M. Kristensen, A.

6. V. Lavrinenko, G. Moulin, H. Ou, C. Peucheret, B. Zsigri, and A. Bjarklev, Nature 441, 199 (2006).

7. J. D. Joannopoulos, S. G. Johnson, J. N. Winn, and R. D.

8. Meade, Photonic Crystals: Molding the Flow of Light,

9. 2nd ed. (Princeton University, 2008).

10. 7. L. H. Frandsen, A. V. Lavrinenko, J. FagePedersen, and P. I.

11. Borel, Opt. Express 14, 9444 (2006).

12. 8. J. Li, T. P. White, L. O'Faolain, A. GomezIglesias, and T. F.

13. Krauss, Opt. Express 16, 6227 (2008).

14. 9. C. Monat, C. Grillet, M. Collins, A. Clark, J. Schroeder, C.

15. Xiong, J. Li, L. O'Faolain, T. F. Krauss, and B. J. Eggleton,

16. Nat. Commun. 5, 3246 (2014).

17. 10. J. G. Pedersen, S. Xiao, and N. A. Mortensen, Phys. Rev. B

18. 78,153101 (2008).

19. 11. R. S. Jacobsen, A. V. Lavrinenko, L. H. Frandsen, C.

20. Peucheret, B. Zsigri, G. Moulin, J. FagePedersen, and

21. P. I. Borel, Opt. Express 13, 7861 (2005).

22. 12. R. Ferrini, D. Leuenberger, R. Houdre, H. Benisty, M. Kamp,

23. and A. Forchel, Opt. Lett. 31, 1426 (2006).

24. 13. T. P. White and A. A. Sukhorukov, Phys. Rev. A 85, 043819

25. (2012).

26. 14. L. O'Faolain, S. A. Schulz, D. M. Beggs, T. P. White, M.
27. Spasenović, L. Kuipers, F. Morichetti, A. Melloni, S.

28. Mazoyer, J. P. Hugonin, P. Lalanne, and T. F. Krauss,

29. Opt. Express 18, 27627 (2010).

30. 15. S. A. Schulz, L. O'Faolain, D. M. Beggs, T. P. White,

31. A. Melloni, and T. F. Krauss, J. Opt. 12, 104004 (2010).

32. 16. A. Gomez-Iglesias, D. O'Brien, L. O'Faolain, A. Miller, and T. F. Krauss, Appl. Phys. Lett. 90, 261107 (2007). 